\title{
POLAR GENERATION OF RANDOM VARIATES WITH THE $t$-DISTRIBUTION
}

\author{
RALPH W. BAILEY
}

\begin{abstract}
The "polar" method of Box and Muller uses two independent uniform variates in order to generate two independent normal variates. It can be adapted so that two variates from Student's $t$-distribution with parameter $\nu$ are generated, though the two variates are now not independent. An algorithm based on the polar method is exact, inexpensive, and valid for all $\nu>0$.
\end{abstract}

Box and Muller's [1] polar method for generating random normal variates relies on two convenient properties of the normal distribution, which we may formulate as follows:

(i) Let $X \sim N(0,1)$. Then $X$ can be regarded as the real part of a complex random variable $Z$ which has a radial distribution (the contours of the density function of $Z$ form circles centered at the origin);

(ii) Write $Z=X+i Y=R e^{i \Theta}$. Then the distribution function $F_{R}\left(\equiv 1-G_{R}\right)$ of $R$ is a simple algebraic expression, so simple that it is invertible; that is, given $G \equiv G_{R}(r)$, we can write down a closed expression for $r$ in term of $G$.

The aim of this article is to show that properties (i) and (ii) are shared by the Student $t$-distribution with parameter $\nu$ (the $t_{\nu}$-distribution) defined by the density

$$
f_{T}(x)=B(\nu / 2,1 / 2)^{-1} \cdot \nu^{-1 / 2} \cdot\left(1+x^{2} / \nu\right)^{-(\nu+1) / 2} .
$$

Thus, we are asserting that the $t_{\nu}$-distribution, like the normal, has a tractable radial parent. If $T$ has the density (1), we shall write " $T \sim t_{\nu}$ ".

Many methods have been proposed for the generation of $t_{\nu}$-variates. The most important ones are described in Devroye [2, pp. 445-450], whose masterly survey we shall not attempt to emulate. The faster algorithms may require either a comparatively great programming effort, or the expensive recalculation of certain quantities required by the algorithm, whenever $\nu$ is changed. As Devroye notes, problems arise when $\nu$ is small and the departure from normality is greatest, particularly in the region $0<\nu<1$, where many of the algorithms fail to work at all.

So one would be interested in a theoretically simple and practically effective generator, valid for any $\nu>0$. We now show how the polar method may be applied to the $t_{\nu}$-distribution to yield such an algorithm.

Received by the editor December 23, 1992 and, in revised form, May 12, 1993.

1991 Mathematics Subject Classification. Primary 65C10; Secondary 62E15. 
Definition. Let $Z=R e^{i \Theta}$ be a complex random variable such that

(i) $R$ and $\Theta$ are independently distributed;

(ii) $\theta$ is uniformly distributed on $[0,2 \pi)$;

(iii) the probability that $R(=|Z|)$ is greater than $r$ is

$$
G_{R}(r)=\left(1+r^{2} / \nu\right)^{-\nu / 2}, \quad r \geq 0, \nu>0 .
$$

Then we shall say that $Z$ has the radial $t_{\nu}$-distribution

The point, and justification, of this definition is the following theorem:

Theorem 1. If $Z=X+Y i$ has the radial $t_{\nu}$-distribution, then the marginal distributions of $X$ and $Y$ are given by $X \sim t_{\nu}$ and $Y \sim t_{\nu}$. The variates $X$ and $Y$ are not independent.

Proof. By assumption, $\Theta$ has density $1 / 2 \pi$, and $R$ has, independently, the density $f_{R}(r)=-d G_{R} / d r=(r / \nu) \cdot\left(1+r^{2} / \nu\right)^{-\nu / 2-1}$. The transformation $x=r \cos \theta, y=r \sin \theta$ (which has Jacobian $r$ ) shows that the joint density of $X, Y$ is

$$
f_{X, Y}(x, y)=(2 \pi)^{-1} \cdot\left(1+\left(x^{2}+y^{2}\right) / \nu\right)^{-\nu / 2-1} .
$$

Now integrate out $y$ (note that the integrand is an even function of $y$ and let $u=\left(1+y^{2} /\left(\nu+x^{2}\right)\right)^{-1}$, expressing the integral as a multiple of a beta integral $)$ to confirm that the marginal distribution of $f_{X}(x)$ has indeed the required form (1). The result for $Y$ follows by symmetry.

The only complex radial distribution for which $X$ and $Y$ are independent with continuous marginal densities is (Mathai and Pederzoli [5, pp. 9-12]) the zero-mean, equal-variance, zero-covariance bivariate normal. Hence $X$ and $Y$ in Theorem 1 are not independent.

[One way of seeing this directly is to examine the distribution of $Y$ conditional on $X$. The density of this distribution at $y$ is $f_{Y \mid X}(y)=f_{X, Y}(x, y) /$ $f_{X}(x)$, and one finds, using the functions $f_{X}$ and $f_{X, Y}$ given by (1) and (3), that $f_{Y \mid X}(y) \propto\left(1+y^{2} /\left(\nu\left(1+x^{2} / \nu\right)\right)\right)^{-\nu / 2-1}$, so that if we define $V=$ $Y\left((1+1 / \nu) /\left(1+X^{2} / \nu\right)\right)^{1 / 2}$, then $X$ and $V$ will be independent, $X \sim t_{\nu}$, and $\left.V \sim t_{\nu+1}.\right]$

Theorem 1 leads to our main result, the polar method for generating $t_{\nu^{-}}$ variates:

Theorem 2. Let $G, H$ be iid variates, uniformly distributed on $[0,1]$. Let $\boldsymbol{\theta}=2 \pi \cdot H$, let $R=\left(\nu\left(G^{-2 / \nu}-1\right)\right)^{1 / 2}(\nu>0)$, let $X=R \cos \boldsymbol{\theta}$, and let $Y=R \sin \Theta$. Then $X \sim t_{\nu}$ and $Y \sim t_{\nu}$. The variates $X$ and $Y$ are not independent.

Proof. Let $Z$ have the radial $t_{\nu}$-distribution. If we let $G=G_{R}(R)$, where $G_{R}$ is given by (2), then we know that $G$ is uniformly distributed on $[0,1]$. Conversely, if $G$ is uniformly distributed on $[0,1]$ and we take the inverse transformation $R=\left(\nu\left(G^{-2 / \nu}-1\right)\right)^{1 / 2}$, then we know that $Z \equiv R \exp (2 \pi i H)=$ $R e^{\Theta i}$ has the radial $t_{\nu}$-distribution. Now apply Theorem 1 .

Theorem 2 is conceptually the simplest formulation of the polar method for the $t_{\nu}$-distribution. However, further improvements are possible. One can (see for instance Marsaglia and Bray [4]) avoid expensive calculation of the cosine 
by using the fact that if $U+V i$ is uniformly distributed on the unit disk, $W \equiv U^{2}+V^{2}$, and $C \equiv U / \sqrt{W}$, then $W$ and $C$ are independent, $W$ has a uniform $[0,1]$ distribution, and $C$ has the same density as $\cos \theta$, that is, $f_{C}(x)=1 / \pi \sqrt{\left(1-x^{2}\right)},-1<x<1$. We incorporate this modification into our proposed algorithm, and note that $Y$ in Theorem 2 is discarded, because of dependence on $X$.

Polar algorithm for generating $t_{\nu}$-variates.

(a) Generate iid uniform $[0,1]$ variates $U$ and $V$. Replace $U$ by $2 U-1$, $V$ by $2 V-1$.

(b) Define $W \equiv U^{2}+V^{2}$. If $W>1$ return to (a).

(c) Let $C=U / \sqrt{W}, R=\left(\nu\left(W^{-2 / \nu}-1\right)\right)^{1 / 2}, X=R C$. Then $X \sim t_{\nu}$.

In order to sidestep one of the square-root calculations, we can rewrite (c) as (c' ) Let $C^{2}=U^{2} / W, R^{2}=\nu\left(W^{-2 / \nu}-1\right), X=\sqrt{\left(R^{2} C^{2}\right)}$.

Thus, the only expensive steps in the polar algorithm are to calculate $W^{-2 / \nu}$ and one square root, whose sign should be chosen at random.

As Neave [6] pointed out in connection with the original Box-Muller algorithm, care must be taken if the uniform random numbers required by the method ( $G$ and $H$ in Theorem 2 ) are in fact pseudorandom numbers generated by the congruential method, as is currently almost invariably the case in practice. Possible cures are surveyed in Golder and Settle [3]. In particular, one can simply use two congruential generators, to different moduli (the "twosequence method"), to generate $G$ and $H$. Golder and Settle show this to be an effective cure.

\section{ACKNOWLEDGMENT}

The author thanks an anonymous referee for helpful comments made.

\section{BIBLIOGRAPHY}

1. G. E. P. Box and M. E. Muller, A note on the generation of random normal deviates, Ann. Math. Statist. 29 (1958), 610-611..

2. L. Devroye, Non-uniform random variate generation, Springer-Verlag, New York, 1986.

3. E. R. Golder and J. G. Settle, The Box-Muller method for generating pseudo-random normal deviates, Appl. Statist. 25 (1976), 12-20.

4. G. Marsaglia and T. A. Bray, A convenient method for generating normal variables, SIAM Rev. 6 (1964), 260-264.

5. A. M. Mathai, and G. Pederzoli, Characterizations of the normal probability law, Wiley Eastern Limited, New Delhi, 1977.

6. H. R. Neave, On using the Box-Muller transformation with multiplicative congruential pseudo-random number generators, Appl. Statist. 22 (1973), 92-97.

Department of Economics, University of Birmingham, Birmingham B31 4BP, United KINGDOM

E-mail address: R. Bailey@uk. ac.bham (Janet)

R. Bailey@bham.ac.uk (Internet) 